\title{
A Computer Vision Application for Measuring the Deflection in a Two-dimensional View of Reinforced Concrete Beams
}

\author{
Eduardo Jr. Piedad ${ }^{*}$, Barne Roxette Carpio², Kristine Sanchez ${ }^{3}$, Marven Jabian ${ }^{4}$ \\ 1 University of San Jose-Recoletos, Cebu City, Philippines, \\ https://orcid.org/0000-0002-9723-1731 \\ 2,3,4 Mindanao State University-lligan Institute of Technology, Iligan City, Philippines, \\ ${ }^{2}$ https://orcid.org/0000-0001-8543-6804, ${ }^{3}$ https://orcid.org/0000-0002-3932-2790, \\ ${ }^{4}$ https://orcid.org/0000-0003-2531-6549 \\ *Email Correspondence: eduardojr.piedad@usjr.edu.ph
}

\begin{abstract}
A novel computer vision application is developed to measure the deflection of twodimensional (2D) reinforced concrete structural members. Eight beam samples, with dimensions of $160 \mathrm{~mm} \times 150 \mathrm{~mm} \times 1400 \mathrm{~mm}$ are loaded and simulated under a four-point loading test until failure using a reaction framework machine. A camera is fixed at the center front view of the concrete beams to capture the deflection of the samples while testing. In each test, a dial indicator is installed and the maximum deflection is manually recorded. Based on the results, the maximum deflection values recorded by the proposed application obtained an average error of $18.38 \%$ when compared to the manual measured results. This indicates that computer vision-based application can provide a beam-wide scale deflection performance, compared to the traditional point-based deflection reading. This study paves a new possibility of aiding manual measurements of concrete beams and all other structural studies.
\end{abstract}

Keywords: Computer vision, beam deflection, dial gauge, structural members, reinforced concrete, reaction framework

\subsection{Introduction}

Computer vision is transforming data from a still or video camera into either a decision or a new representation (Bradski \& Kaehler, 2008). All such conversions, also known as image processing, are done to be utilized for practical purposes. These include character recognition, blood cell analysis, computer-aided technology (CAT), land-use identification, and traffic monitoring, among others (Vernon, 1991). In this paper, a novel computer vision application is used for testing the structural concrete beams used in buildings. This application aims to support and aid manual inspection and testing to have better and more accurate measurements.

One of the promising benefits of machine vision is measuring the deflection in structural members. Deflection is an important property that must be satisfied by structural members in terms of serviceability requirements. While accurate concrete beam deflection sensors are commercially available such as dial gauges, linear-variable 
differential transformers, and laser displacement sensors (LDS), they are point-wise devices; this implies that they can only measure the deflection in one dimension. Thus, Maas and Hampel (2006) suggested that if two- or three-dimensional measurements are required, point-wise sensors would not be suitable. Hence, studies have been conducted such that of Florczyk (2005), Lü et al. (2013), and Chaczko et al. (2010) that availed the use of machine vision applications in obtaining deflection in 2D or 3D measurements.

This study primarily addresses the need to provide an alternative and innovative option for deflection measurement focusing on laboratorybased testing of structural members. The aims of this study are four-fold: first, to develop a working computer vision application that can measure the deflection; second, to assess the reliability of the application by calibration using optics principles; third, to measure deflection in two-dimension for reinforced concrete structural member samples, subjected to four-point loading in a reaction framework machine; and lastly, to compare the results of the maximum deflection of the application with that of a dial gauge.

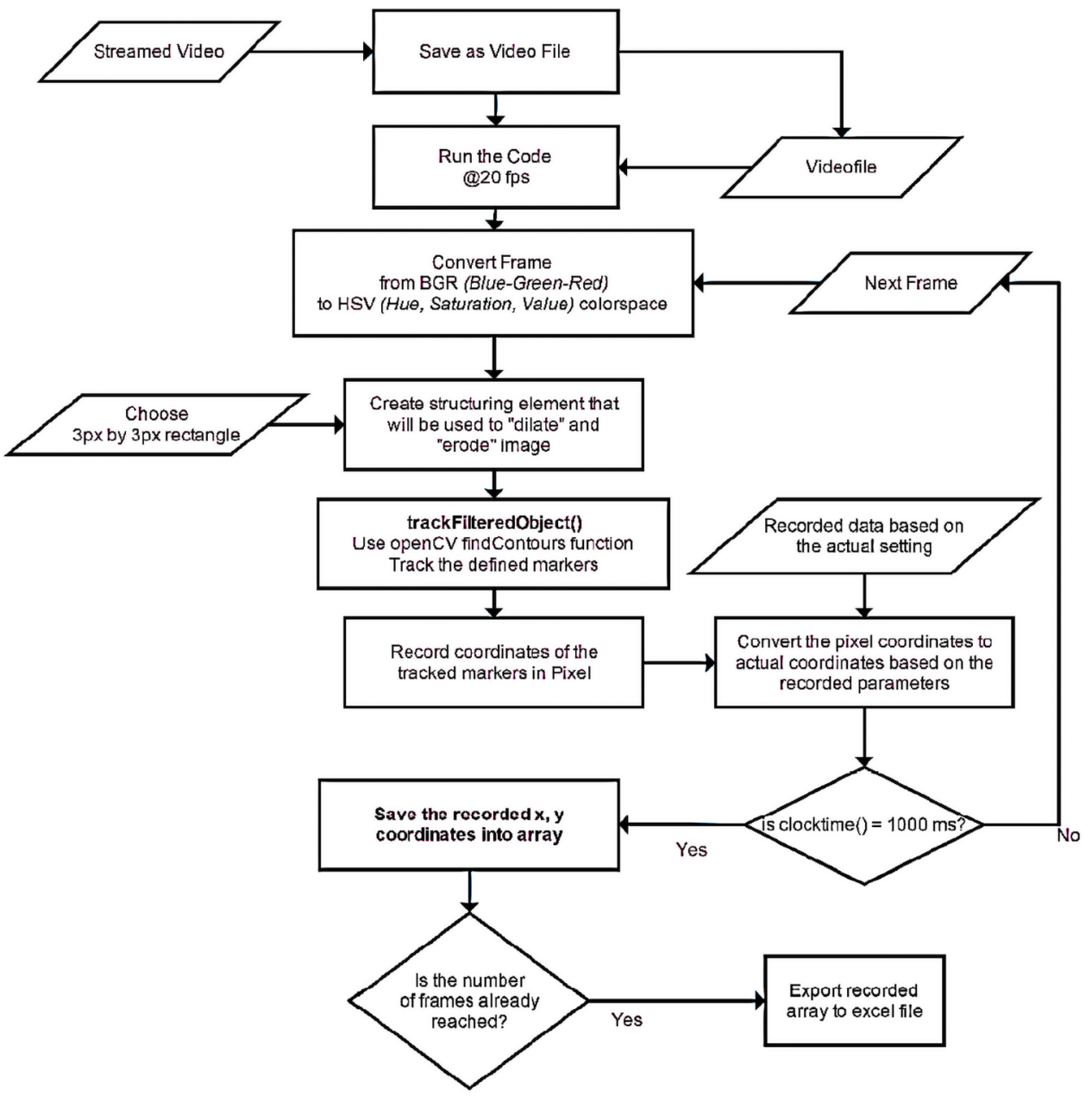

Figure 1. A Proposed Computer Vision Methodology for Beam Deflection Reading 


\subsection{Methodology}

\section{Application Development}

Figure 1 shows the process flowchart. The methodology follows a typical computer vision procedure, as in the works of Piedad et. al (2019), and only adding some process intended for structural studies which is beam deflection measurement. As shown, the video file first records the streamed video before running the code. This process is done to reserve another copy for future debugging. It is also important to note that the code must be optimized in a way that the clocktime() should precisely be equal to 1000 milliseconds. Note that the streaming rate is at 20 frame per second (fps) which is close to human eye cognition.

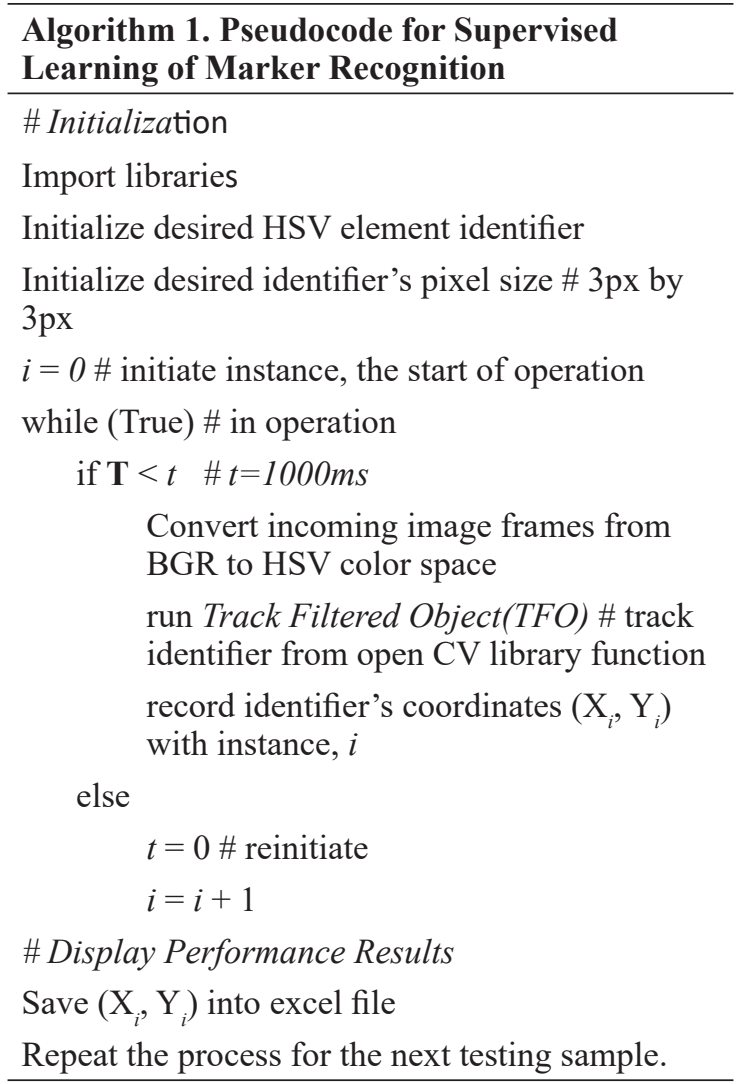

Algorithm 1 presents the pseudocode of tracking the markers using Open CV (Open-Source Computer Vision) libraries. This algorithm is similar to the works of Piedad and Villeta (2016) with a few modifications in the camera settings - distance of the camera to the beam loading, and the identifier's values and initializations. The computer vision application use $\mathrm{C}++$ programming language in Visual Studio 2013 developer environment with Open CV. The actual code of the pseudocode shown in Figure 1 and Algorithm 1 is published in the GitHub website (Piedad, 2015). The developed offline application is run in ACER laptop computer with i3 processor and 4GB RAM memory.

\section{Making the Test Samples}

The researchers made eight reinforced concrete beam samples with dimensions $160 \mathrm{~mm} x$ $150 \mathrm{~mm} \times 1400 \mathrm{~mm}$. Before making the samples, the study conducted quality tests for both the fine and coarse aggregates. The tests include sieve analysis, specific gravity, and absorption test. The study used the guidelines from the American Society for Testing and Materials (ASTM) International standards for the C127-15 (ASTM International, 2001), C192-C192M-02 (ASTM International, 2002), and C128-01 (ASTM International, 2003) tests. The fine aggregates used river sand, while the coarse aggregates employed $3 / 4$ inch crushed stone. The study also used an ordinary Type I Portland cement, with a water-cement ratio kept at 0.47 . For the quality of the concrete mixture, the researchers conducted a slump test to ensure the workability of the mix.

After mixing, the researchers placed the samples in a curing tank filled with water for 28 days to attain the specified concrete compressive strength of 44.2 Megapascals (MPa). Figure 2 shows the photograph of the samples. 


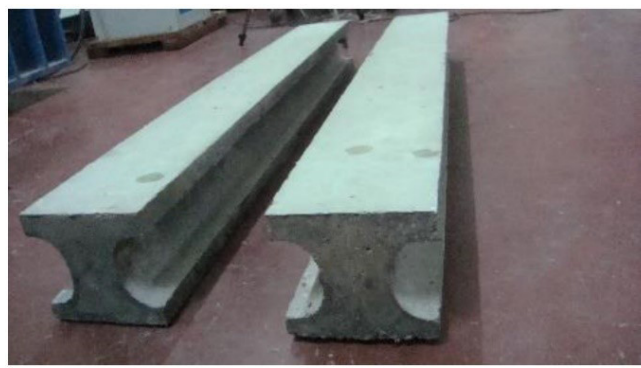

(a)

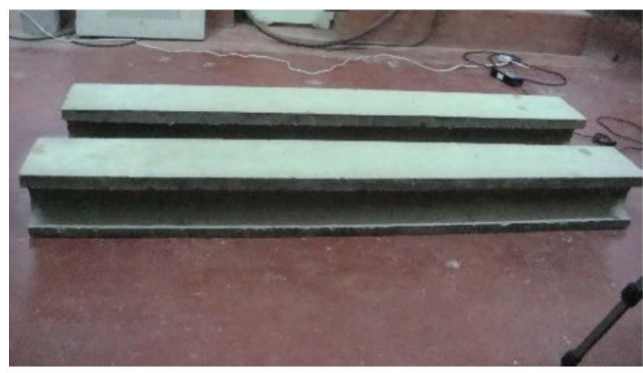

(b)

Figure 2. Two Reinforced Concrete Beam Samples Used in the Study

After mixing, the researchers placed the samples in a curing tank filled with water for 28 days to attain the specified concrete compressive strength of 44.2 Megapascals (MPa). Figure 2 shows the photograph of the samples.

\section{Calibrating the Computer Vision Application Using Optical Principles}

In this study, markers had been attached to the samples to indicate deflection. Hence, it is important to calibrate the actual angle of tilt of the markers, $\alpha_{\mathrm{am}^{\prime}}$, with the theoretical angle of tilt, $\alpha$. The researchers used the principle of optics for calibration. As shown in Eq. (1), the angle $\alpha$, in radians, can be obtained as:

$$
\alpha=\tan ^{-1} \frac{y}{x}
$$

where $y$ is the vertical distance from the marker to the lens, and $\mathrm{x}$ is the horizontal distance.

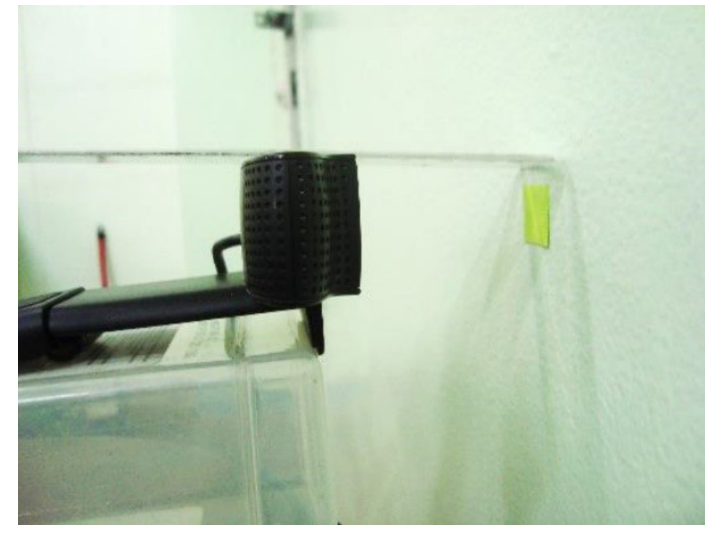

Figure 3. Center Marker Aligns to the Center Axis

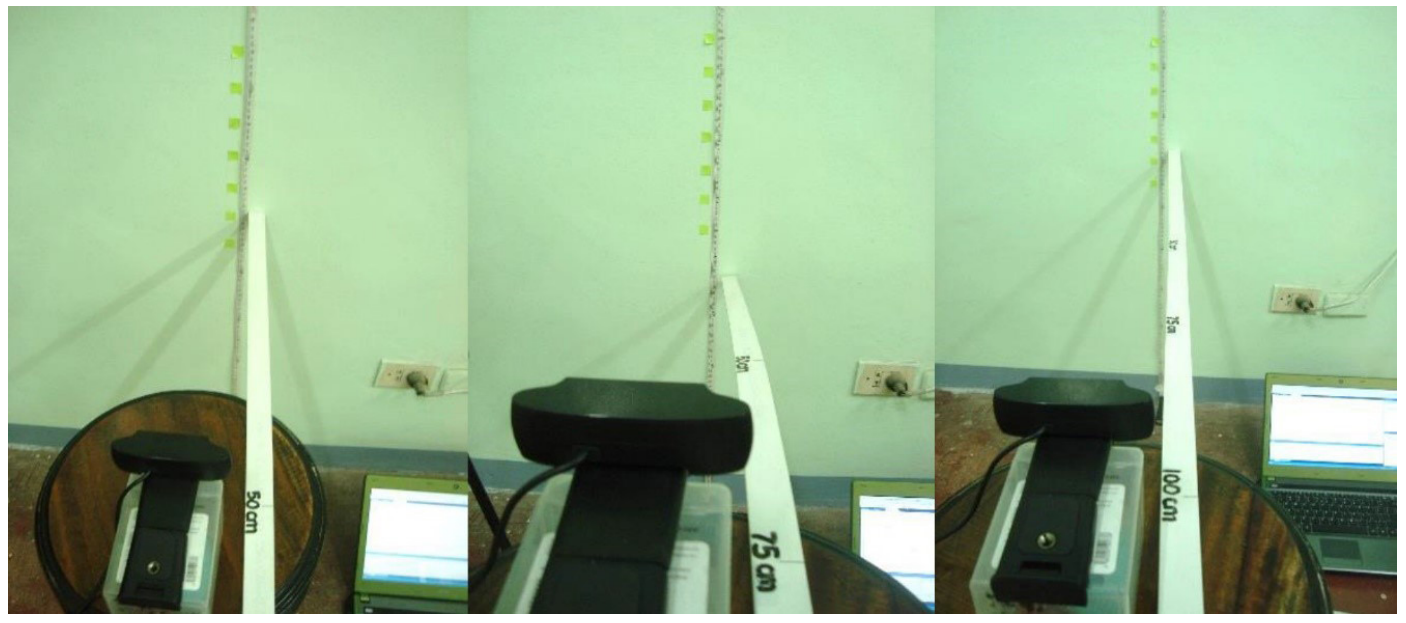

Figure 4. Optical Calibration Test of 500, 750- and 1000-mm Distances 


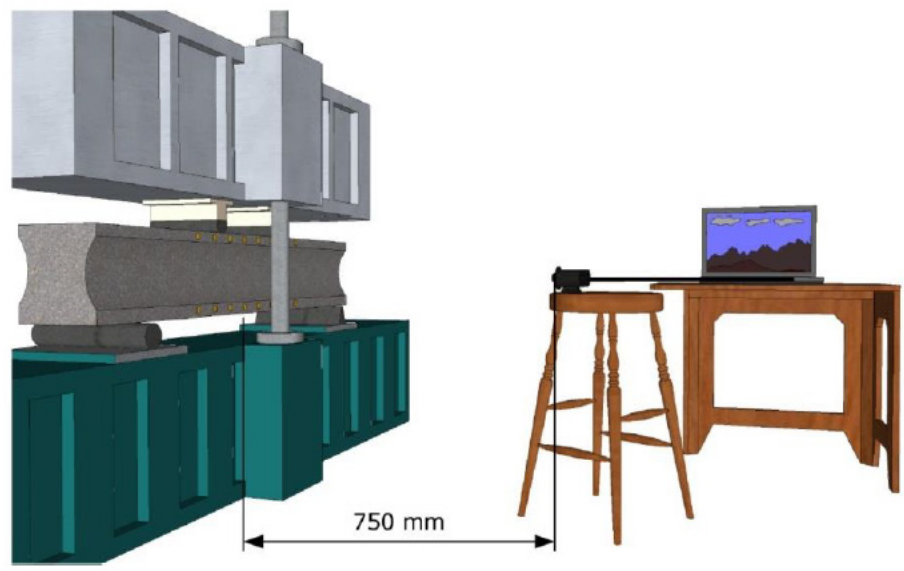

(a)

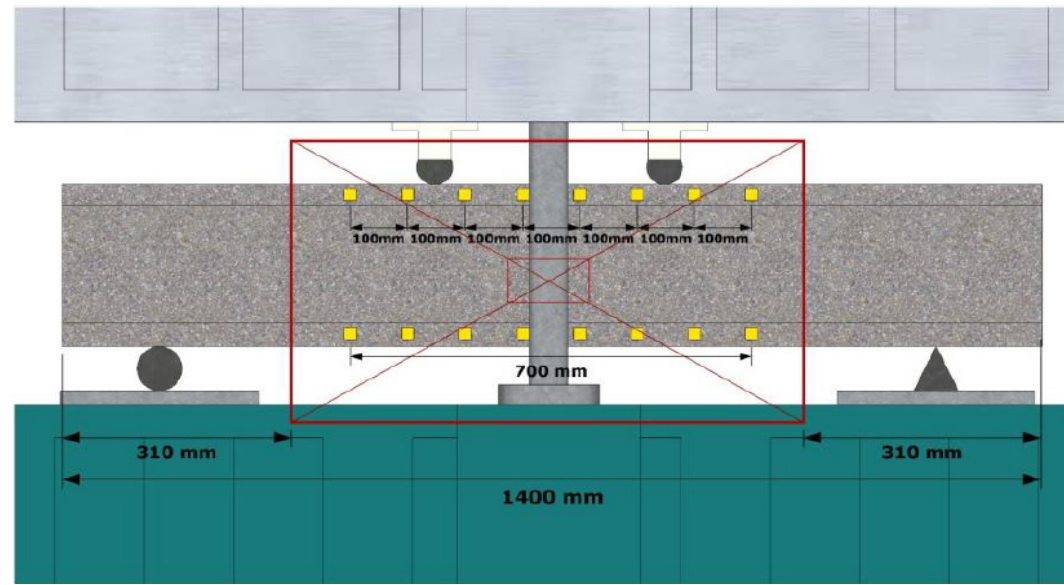

(b)

Figure 5. (a) Left Side View, (b) Front View of the Setup

The researchers conducted the calibration under a three lens-to-object (LTO) distances of 500, 750, and 1000 millimeters (Piedad \& Villeta, 2016). Figures 3 and 4 show the photographs taken during calibration.

\section{Calibrating the Application for Marker Recognition}

Before the experimental setup, the researchers conducted the calibration test of computer vision application. This test assured the recognition of the markers to the application. The HSV filter, also known as color-detection, determines the minimum and maximum values of Hue Saturation Value.

\section{Laboratory Testing and Measuring Deflection of} the Test Samples

The application used a single available Logitech c920 webcam taking the two-dimensional (2D) view of the test subjects. The camera is positioned in a fixed-center front view, as shown in Figures 5(a) and 5(b). 
The test samples were loaded until they failed under four- point loading using the reaction force framework machine. The machine recorded the maximum loads just before the failure occurred. The study employed a dial indicator to determine the maximum deflection at the mid-span. The values given by the dial indicator was used to calibrate the prototype. Also, the camera captured the deflection of the beam with the use of the markers. Finally, the researchers compared and evaluated the two results from the prototype and the dial indicator.

\subsection{Results}

Graph of Deflection Values from the CV Application in Two-dimension

Figure 6 (a)-(h) illustrate the deflection of the beam as captured by the camera using the attached markers. The elevations of the top and bottom portions of the beam, in the initial state (un-deflected) and final state (deflected) just before it cracked, are plotted for the length of the beam within the camera frame. At every $100 \mathrm{~mm}$ interval, changes in elevation on the beams were recorded through the markers.

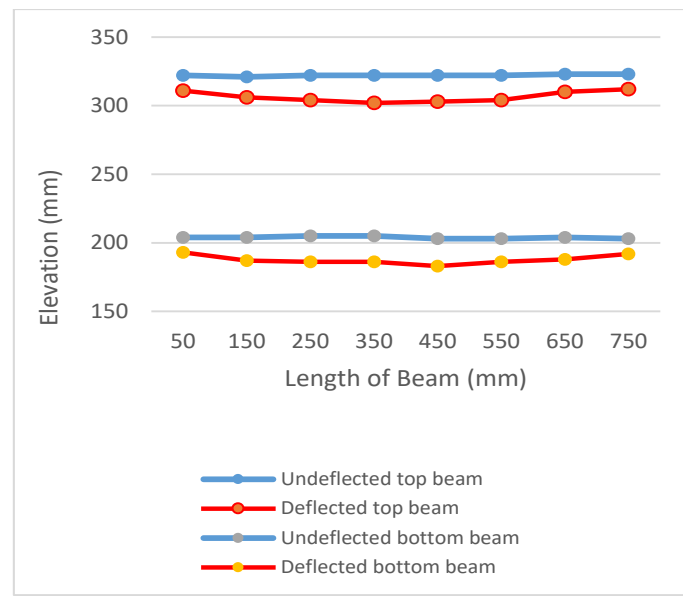

(a)

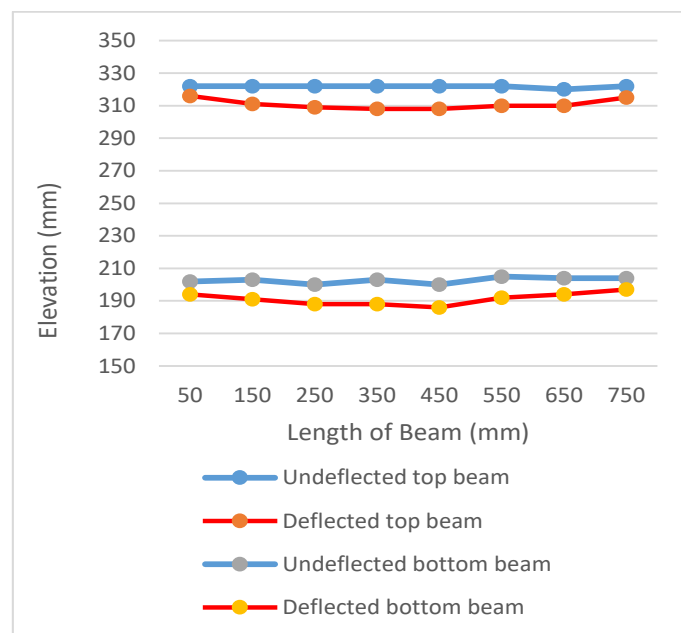

(c)

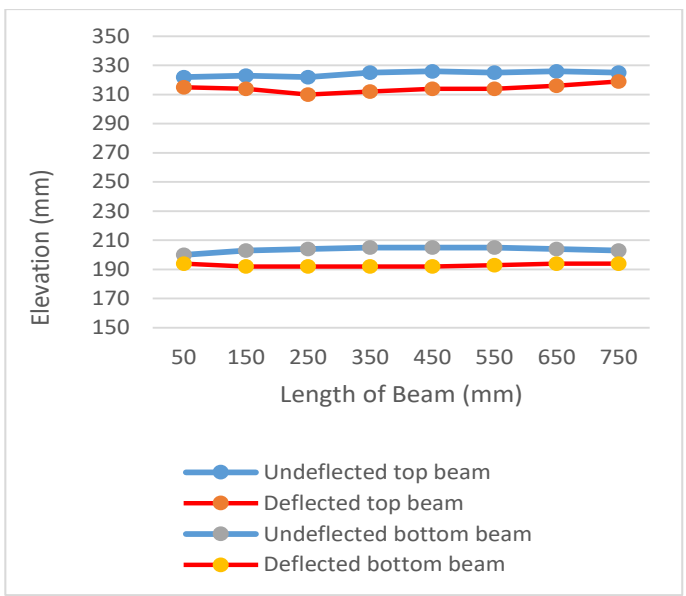

(b)

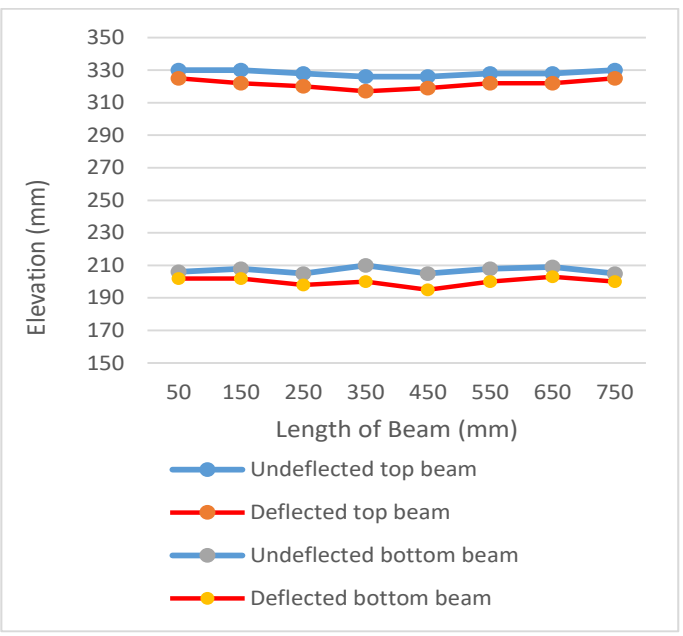

(d)

Figure 6. (a)-(d). Graph of the Initial and Deflected Forms of the Concrete Beam Amples 


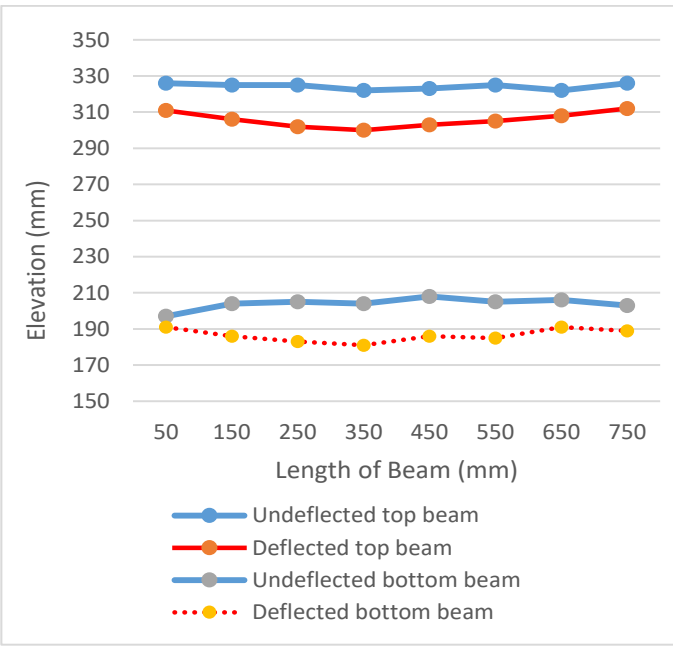

(e)

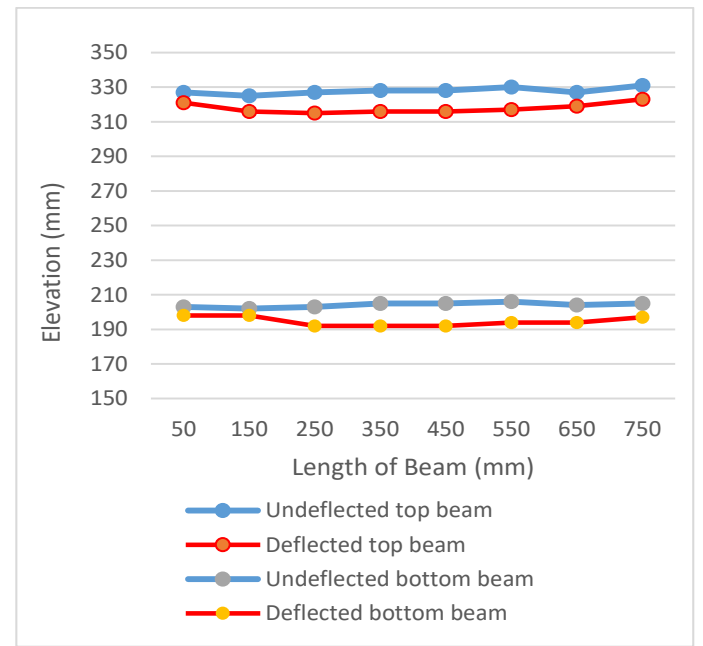

(g)

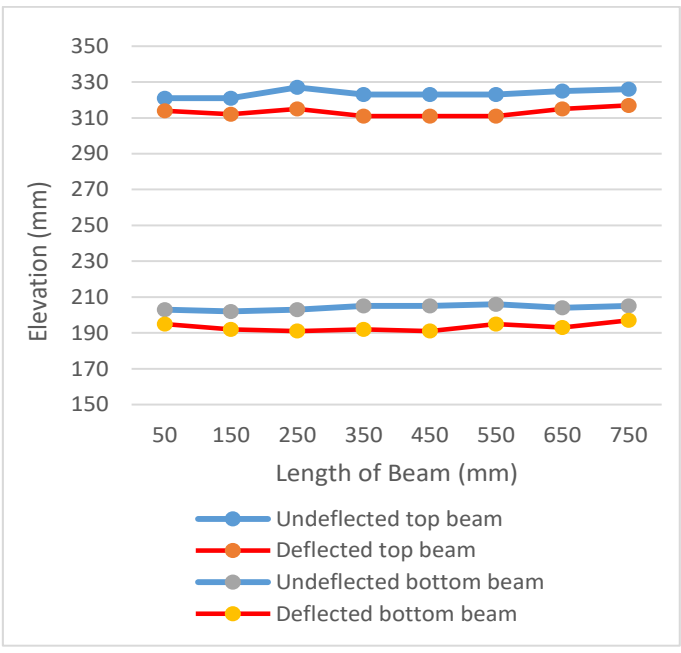

(f)

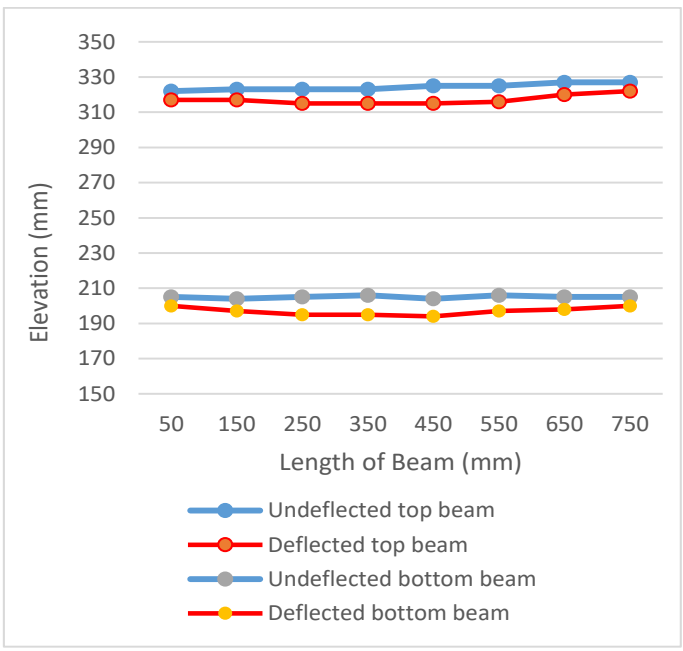

(h)

Figure 6. (e)-(h). Graph of the Initial and Deflected Forms of the Concrete Beam Amples

The deviations of the actual initial state are due to the error read in the markers as calibrated from the optical principle; hence, the un-deflected state is not always a straight line. However, the study successfully obtains the deflection of the beam samples in a two-dimensional view during testing since, all the graphs in Figure 6 show a downward change in elevation from its initial state to its final form.

\section{Comparison of the Results with a Dial Indicator}

Table 1 shows the values of maximum deflection obtained from the computer vision (CV) application and the dial indicator. Two results can be obtained from the CV application namely; the upper and the lower deflections of the beam. Figure 7 shows the setup. 
Table 1. Maximum Deflection Values $(\mathrm{mm})$

\begin{tabular}{|c|c|c|c|c|}
\hline \multirow{2}{*}{$\begin{array}{c}\text { Sample } \\
\text { No. }\end{array}$} & \multicolumn{2}{|c|}{ CV application } & \multirow{2}{*}{$\begin{array}{c}\text { Dial } \\
\text { indicator }\end{array}$} & \multirow{2}{*}{ Error, \% } \\
\hline & Upper & Lower & & \\
\hline 1 & 20 & 20 & 17 & 17.65 \\
\hline 2 & 13 & 13 & 14 & 7.14 \\
\hline 3 & 15 & 15 & 14 & 7.14 \\
\hline 4 & 10 & 9 & 13 & 26.92 \\
\hline 5 & 23 & 23 & 21 & 9.52 \\
\hline 6 & 14 & 12 & 18 & 27.78 \\
\hline 7 & 14 & 12 & 18 & 27.78 \\
\hline 8 & 10 & 10 & 13 & 23.08 \\
\hline
\end{tabular}

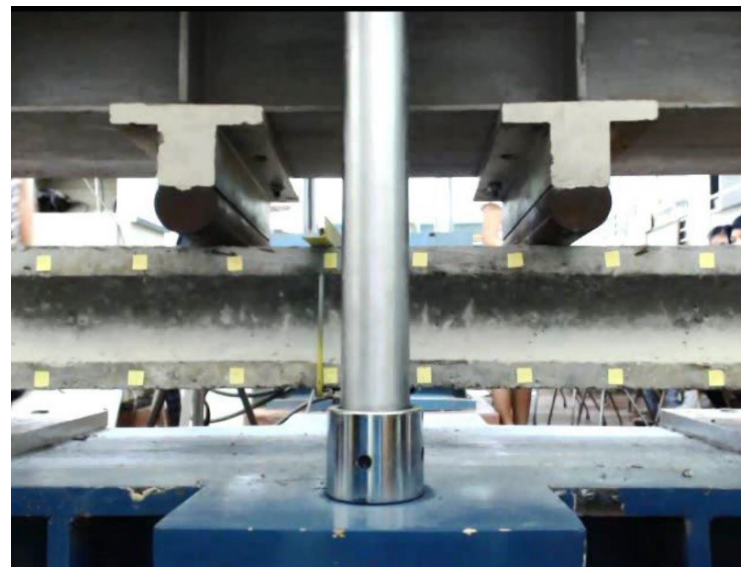

Figure 7. Actual Camera View with Markers

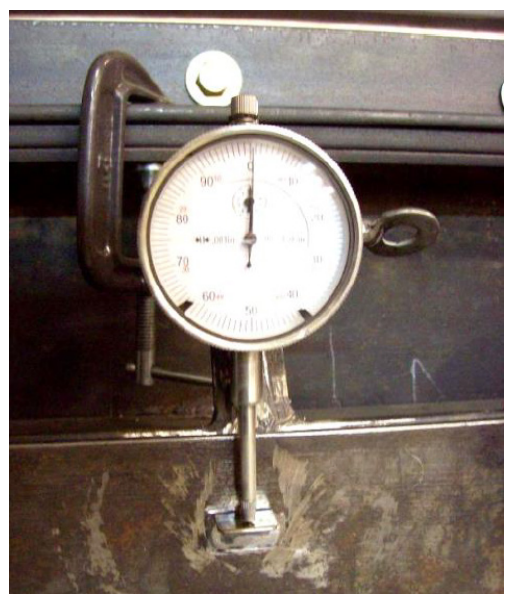

Figure 8. Dial Indicator
Figure 8 shows a dial indicator. One full revolution of the dial represents $1 \mathrm{~mm}(0.0394$ inches). These indicators measure angular displacement and not linear displacement. Linear distance correlates to the angular displacement based on the correlating variables. If the cause of movement is perpendicular to the finger, the linear displacement error is acceptably small and within the display range of the dial.

When the deflection values from the CV application were compared from the dial indicator, it turned out, errors were observed ranging from $7.14 \%$ to $27.78 \%$, with an average percent of 18.38 $\%$. The result indicates that the CV application provides a good result when obtaining the deflection compared to the dial indicator.

\subsection{Conclusion}

This study developed a computer vision application. It measures the deflection in a twodimensional view of reinforced concrete beams in a controlled environment. The study performed a calibration to assess the reliability of the application, which provided a $1.51 \%$ error of the angle of tilt when compared to the theoretical angle of tilt. Due to the error in the angle of tilt, the graph deviated from its expected straight line. However, the application successfully graphed the deflection of the beams in a two-dimensional view since, the deflected state of the beams exhibited a downward change in elevation from the undeflected state. Also, the result from the computer vision application obtained an $18.38 \%$ average error when compared with the dial indicator.

The study recommends a comparison of the results with the theoretical deflection values of the beam using double integration method. The equation obtained shows the following: the deflection is a function of the applied load; the unsupported span of the beam; the modulus of elasticity of concrete; the moment of inertia; 
and the distances from the supports. However, the study was unable to determine the complete results of the physical properties. Hence, only the results of the dial indicator served as the means for comparison of the deflection values with that of the computer vision application. Overall, this potential application can provide a beam-wide scale and time-based deflection performance compared to the conventional point-based deflection reading.

\section{References}

American Society for Testing and Materials, International. (2001). Standard test method for density, relative density (specific gravity), and absorption of coarse aggregate, C127-15. ASTM International. 10.1520/C0127-15

American Society for Testing and Materials, International. (2002). Standard practice for making and curing test specimens in the laboratory, C192-C192M-02. ASTM International. 10.1520/C0192_C0192M-02

American Society for Testing and Materials, International. (2003). Standard test method for density, relative density and absorption of fine aggregate, C128-01. ASTM International. 10.1520/C0128-01E01

Bradski, G.,\&Kaehler, A. (2008). Learning OpenCV: Computer vision with the OpenCV library. O'Reilly Media, Inc.

Chaczko, Z., Yeoh, L. A., \& Mahadevan, V. (2010, February 9-11). A preliminary investigation on computer vision for telemedicine systems using OpenCV [Conference session]. Second International Conference on Machine Learning and Computing, Bangalore, India. 10.1109/ICMLC.2010.70
Florczyk, S. (2005). Robot vision: Video-based indoor exploration with autonomous and mobile robots. WILEY-VCH Verlag GmbH \& Co. KGaA.

Lü, C., Wang, X., \& Shen, Y. (2013, December 1618). A stereo vision measurement system based on OpenCV [Conference session]. 6th International Congress on Image and Signal Processing, Hangzhou, China. 10.1109/ CISP.2013.6745259

Maas, H. G., \& Hampel, U. (2006). Photogrammetric techniques in civil engineering material testing and structural monitoring. Photogrammetric Engineering \& Remote Sensing, 72(1),39-45. https://www.asprs.org/wp-content/uploads/ pers/2006journal/january/2006_jan_39-45. pdf

Piedad, E. J. (2015). Civil-ivision. https://github.com/ epiedadjr/civil-ivision.git

Piedad, E.D. Jr., \& Villeta, R. B. (2016). Displacement and illumination levels effect on shortdistance measurement errors of using a camera. Recoletos Multidisciplinary Research Journal, 4(1). https://doi.org/10.32871/ rmrj1604.01.06

Piedad, E. J., Le, T. T., Aying, K., Pama, F. K., \& Tabale, I. (2019, October 17-20). Vehicle count system based on time interval image capture method and deep learning mask R-CNN [Conference session]. TENCON 2019-2019 IEEE Region 10 Conference (TENCON), Kochi, India. 10.1109/ TENCON.2019.8929426

Vernon, D. (1991). Machine vision: Automated visual inspection and robot vision. Prentice-Hall International. 